\title{
VIBRATION ANALYSIS OF THE FLEXIBLE BEAM USING DYNAMIC SOLVER K_SIM
}

\author{
Dong Il Park ${ }^{1 *}$ and Doohyung Kim² \\ Department of Robotics and Mechatronics, Korea Institute of Machinery \& Materials, Korea parkstar@kimm.re.kr,
}

\section{*Corresponding Author: -}

Email: parkstar@kimm.re.kr

\begin{abstract}
: -
We developed the dynamic solver including the pre-processor with GUI, kinematic/dynamic solver and the post-processor. This can support to analyze the flexible body dynamics as well as the rigid body dynamics. Because almost robot system has the multi bodies including some flexible bodies, multi flexible body dynamics is very important. In the paper, we carried out the vibration analysis of the flexible beam using the developed dynamic solver $K_{-}$Sim and compared it to the commercial multi flexible body dynamic solver.
\end{abstract}

Keywords: - dynamic solver, K_Sim, Flexible beam, Beam element 


\section{INTRODUCTION}

The mechanical systems such as a robot, a machine tool, a vehicle and so on generally have multi bodies and multi joints. It is important to predict the motion and the interaction forces during the stage of the system design. Dynamic simulation for the flexible multi-body system is carried out including the deformation and the stress.

There are many researches about the dynamic simulation methodology for the flexible multi-body system.[1]-[5] We also developed the dynamic solver including the pre-processor with GUI, kinematic/dynamic solver and the post-processor as Fig. 1.[6]-[7] This can support to analyze the flexible body dynamics as well as the rigid body dynamics. Because almost robot system has the multi bodies including some flexible bodies, multi flexible body dynamics is very important. We have performed the dynamic simulation of the substrate handling robot with the flexible end-effector using the commercial software.[8]-[9] In the paper, we carried out the vibration analysis of the flexible beam using the developed dynamic solver K_Sim and compared it to the commercial multi flexible body dynamic solver.

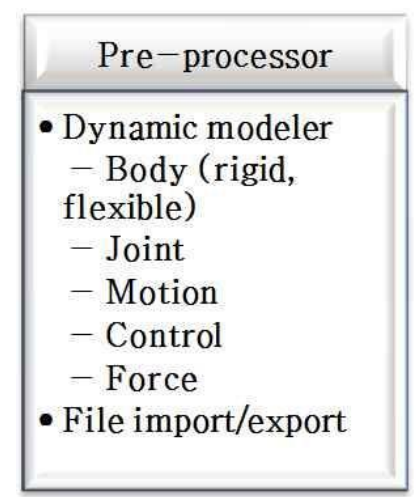

\begin{tabular}{|l|}
\hline \multicolumn{1}{|c|}{ Solver } \\
\hline - Kinematic/Dynamic \\
Solver \\
- Rigid body \\
dynamics \\
- Flexible body \\
dynamics \\
- With \\
control/optimization \\
\hline
\end{tabular}

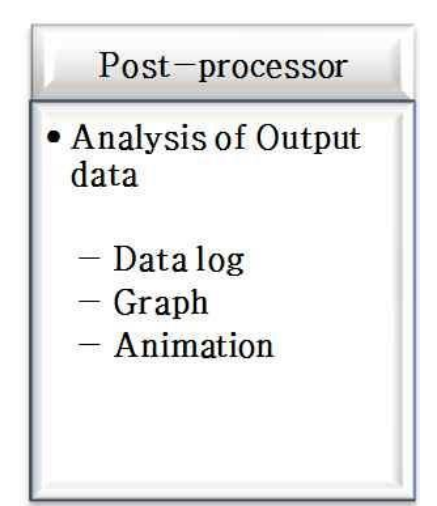

Fig. 1 The developed dynamic solver K_Sim

\section{Modeling Of The Robot}

Fig. 2 shows the substrate handling robot with the flexible body. It's end-effector was made of CFRP material because the robot end-effector has to be light. The robot model is simplified as Fig. 3 for the dynamic simulation and all components including the rigid parts, the flexible parts, joints and motions are represented as TABLE I.

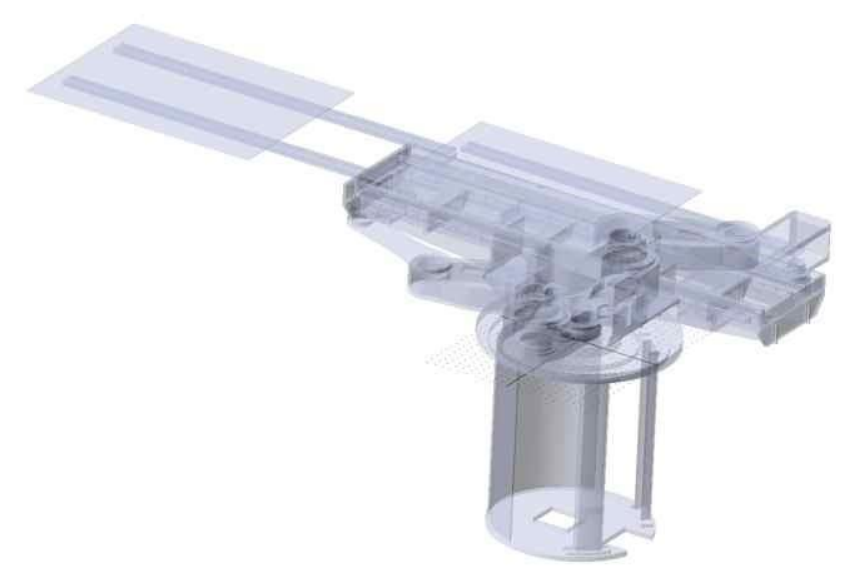

Fig. 2 The substrate handling robot with the flexible body

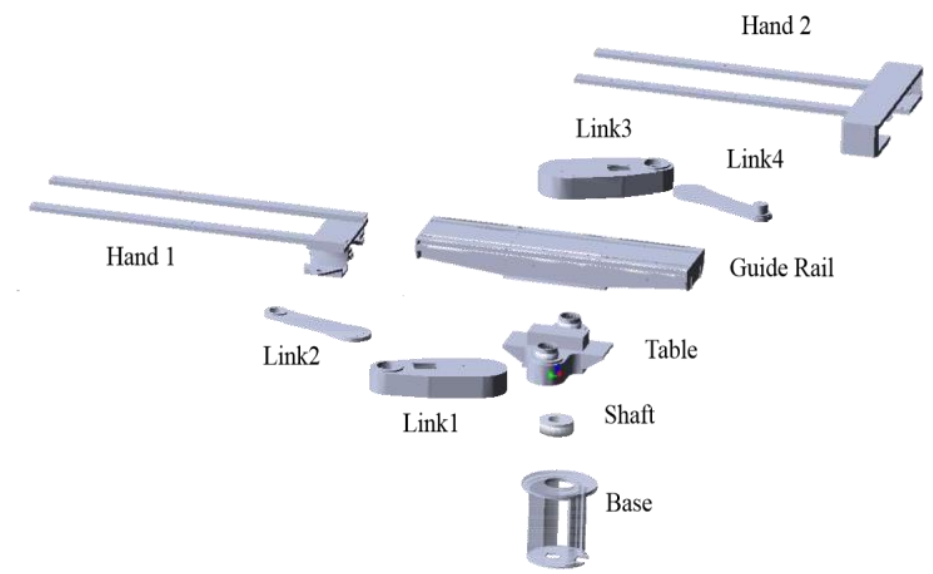

Fig. 3 All parts of the simplified robot model for the simulation 
TABLE I The lists of the parts, joint and motion

\begin{tabular}{|c|c|c|}
\hline \multirow{2}{*}{ Part(Rigid) } & & Base \\
\cline { 3 - 3 } & \multirow{2}{*}{7} & Shaft \\
\cline { 3 - 3 } & & Table \\
\cline { 2 - 3 } & & Link 1, 2, 3,4 \\
\hline Part (Flexible) & 2 & Hand 1, 2 \\
\hline Part (payload) (w/wo) & 2 & Plate 1, 2 \\
\hline Revolute Joint & 3 & \\
\hline Translational Joint & 3 & \\
\hline Bracket Joint & 4 & \\
\hline Spherical Joint & 2 \\
\hline Universal Joint & 2 \\
\hline Motion & 4 \\
\hline
\end{tabular}

The robot base is fixed on the ground and the shaft is connected to the base by the translational joint to moves vertically. There is a revolute joint between the shaft and the table. There are some more revolute joints among the table, the link1, the link 2 and the hand 1 and the structure of the opposite side is same. Some revolute joints have to be converted to the universal joint or the spherical joint because the table, the link1, the link 2 and the hand 1 is composed by 4-bar mechanism and it must have the available DOF for simulation. Fig. 4(a) is the designed template with only rigid body and Fig. 4(b) is the designed template with the flexible body. The control template is also composed as Fig. 5.
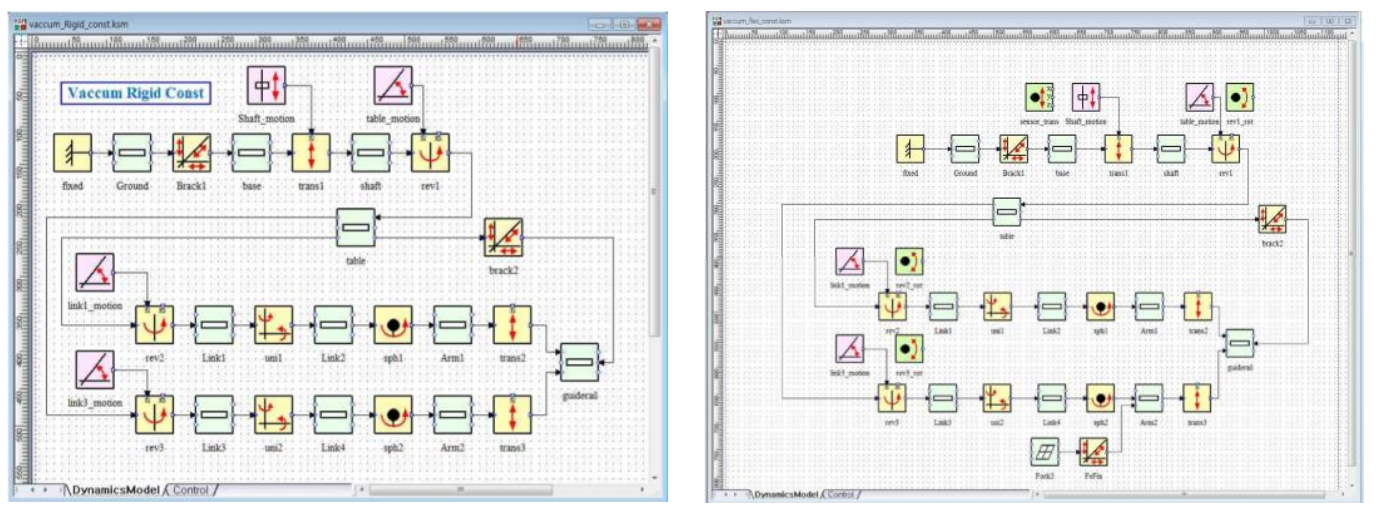

(a) K_Sim_Modeler template with only rigid body (b)K_Sim_Modeler template with the flexible body Fig. 4 K_Sim_Modeler template of the robot

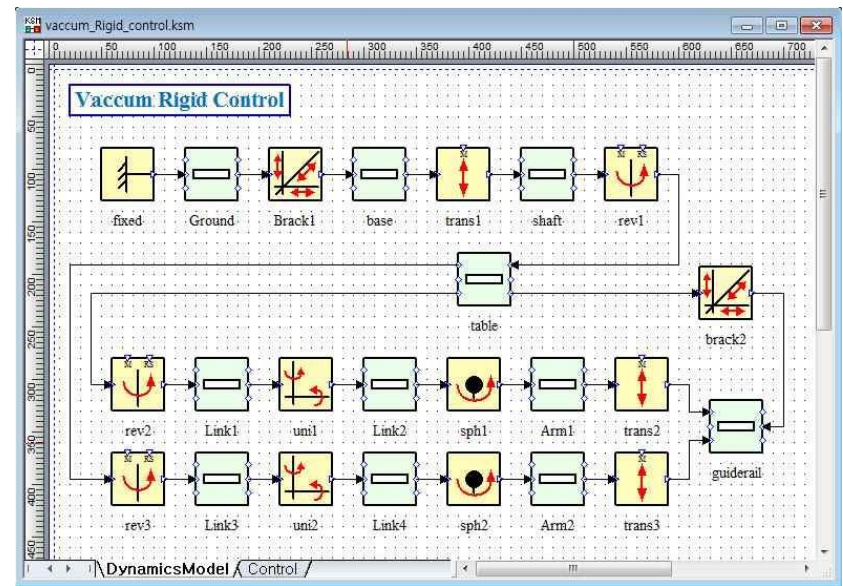

Fig. 5 K_Sim_Modeler template with control

The hand 1 and the hand 2 are modeled as the flexible body components as mentioned above and they are analyzed as the simplified flexible beam model as Fig. 6. One side of the hand is fixed on the rigid link and the other side is free. If there is a plate on the hand, the distributed mass are applied to each element. 


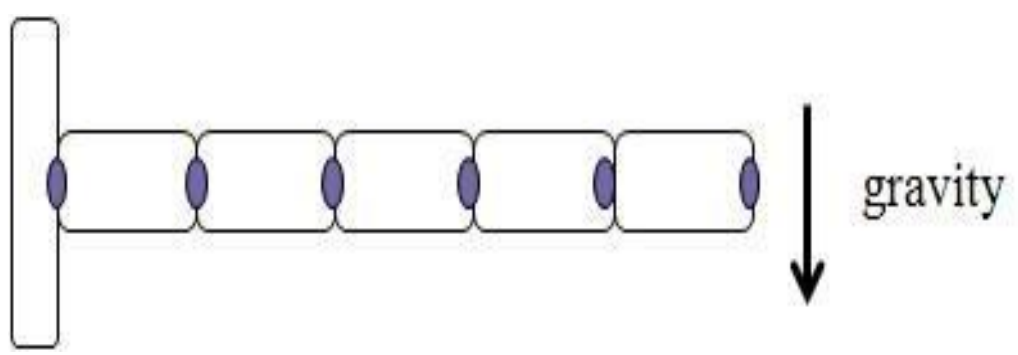

Fig. 6 The simplified model of the flexible beam

\section{Analysis Of The Rigid Body}

The designed model template is applied to the solver and the dynamic simulation is carried out on the various conditions. The motion plan used in the dynamic simulation was based on the motion in the real manufacturing line. We looked into the vibration of the end-effector and compared the results with the one of the commercial solver as Fig. 7. The result for the position and the torque is almost similar and K_Sim proved to have good performance $t$

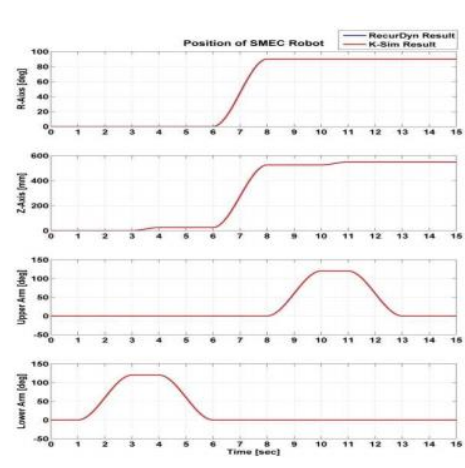

(a) Comparison of the result : Position

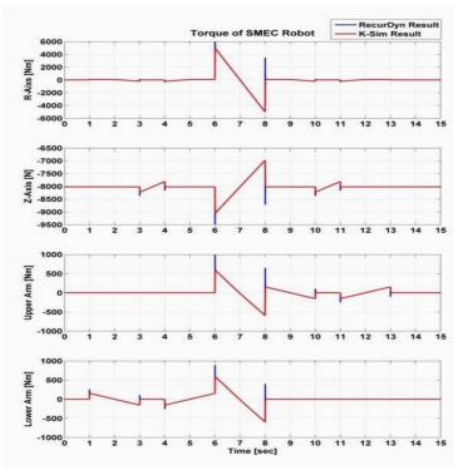

(b) Comparison of the result : Torque

Fig. 7 Comparison of the result

\section{Analysis Of The Flexible Body}

As the next step, the designed template with the flexible body is also applied to the solver and the simulation is carried out. Fig. 8 shows the static simulation result for the flexible beam and it is proved to get the exact result.

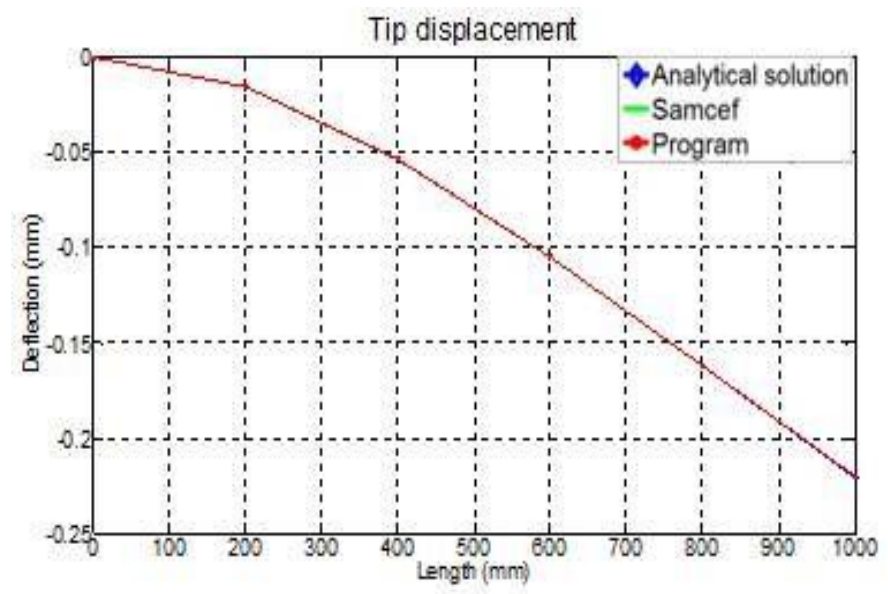

Fig. 8 Comparison of the result

The dynamic simulation is also carried out and the condition at the dynamic simulation condition is same with the one at the rigid body simulation. We could obtain the vibration of the end-effector as Fig. 9 and its validity is proved with the comparison with the commercial software. 


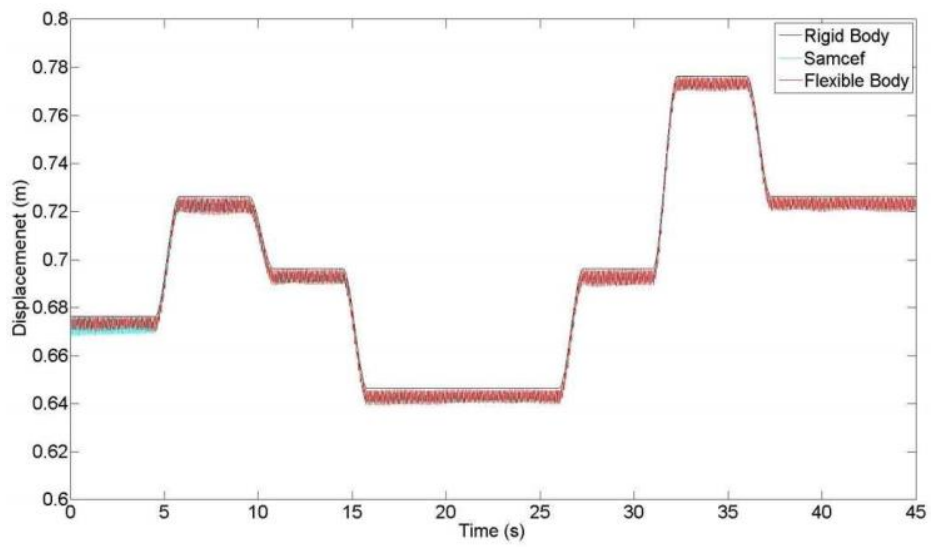

Fig. 9 Comparison of the result (Dynamic simulation)

\section{Conclusion}

We developed the dynamic solver including the pre-processor with GUI, kinematic/dynamic solver and the post-processor in the previous research. This solver can support to analyze the flexible body dynamics as well as the rigid body dynamics. In the paper, we carried out the vibration analysis of the flexible beam using the developed dynamic solver $\mathrm{K} \_$Sim and compared it to the commercial dynamic solver. The motion simulation for many kinds of the flexible multi-body systems can be conveniently performed with the developed dynamic solver K_Sim to analyze the motion, the interaction forces, the stress, the deformation and so on.

\section{Acknowledgement}

This work was supported by the R\&D program of Korea government.

\section{References}

[1].F. Schivao, G. Ferretti and L. Vigano, Object-Oriented Modelling and simulation of flexible multibody thin beams in modelica with the finite element method, Modelica 2005, pp. 25-34, 2005.

[2].F. Resta, F. Ripamonti, G. Cazzulani and M. Ferrari, Independent modal control for nonlinear flexible structures: an experimental test rig, Jounal of Sound and Vibration, vol. 329, pp. 961-972, 2010.

[3].Jorge Martins, Miguel A. Botto,Jose S. D. Costa, "Modeling of Flexible Beams for Robotic

[4].Manipulators," Multibody System Dynamics, vol. 7, pp. 79-100, 2002

[5].X. Murua, F. Martinez, A. Pujana, J. Basurko, Juan M. Pagalday, "3D Flexible Multibody Thin Beam simulation in Modelica with the Finite Element Method," Modelica 2006, pp. 97-105, 2006 [5] Wook Hyeon Kim, Tae Won Park, Dong Il Park, "Development of flexible mutibody dynamics program for beam type solar cell manipulator," The 6th Asian Conference on Multibody Dynamics, 2012, pp. 1-6

[6].Wook Hyeon Kim, Tae Won Park, Dong Il Park, "Vibration control of a flexible fork of a solar cell manipulator for accurate positioning in large vertical motion," ECCOMAS Multibody Dynamics 2013, Croatia, pp. 203-204

[7].Dong Il Park, Cheol Hoon Park, "Motion Simulation of Solar Cell Substrate Handling Robot," 2010

[8].Second IITA International Joint Conference on Artificial Intelligence (JCAI 2010), Paper No.809, Dec., 2010, Shanghai, China

[9].Dong Il Park, Cheolhoon Park, Yijun Yoo, Hyunmin Do and Jin-Ho Kyung, "Dynamic analysis of beam type substrate handling robot in solar cell manufacturing," The 8th International Conference on Ubiquitous Robots and Ambient Intelligence, pp. 794-795

[10]. Cheol Hoon Park, Dong il Park and Hyun Min Do, "Controller Design and Motion Simulation of

[11]. Solar Cell Substrate Handling Robot in Vacuum Environment," 2011 11th International Conference on

[12]. Control, Automation and Systems, pp.1017-1019, Oct., 2011, Gyeonggi-do, Korea 